\title{
Modelling Swelling, Erosion and Release of Drug from Hydrogel Based Solid Matrices
}

\author{
G. LAMBerti ${ }^{1}$, I. GALDI ${ }^{1}$, G. Titomanlio ${ }^{1}$, A. A. BARbA ${ }^{2}$, M. D'AMORE ${ }^{2}$ \\ ${ }^{1}$ Dipartimento di Ingegneria Chimica e Alimentare, Università di Salerno, Fisciano, Italia \\ ${ }^{2}$ Dipartimento di Scienze Farmaceutiche, Università di Salerno, Fisciano, Italia \\ E-mails: glamberti@unisa.it (G. Lamberti), igaldi@unisa.it (I. Galdi), gtitomanlio@unisa.it (G. Titomanlio), \\ aabarba@unisa.it (A. A. Barba), mdamore@unisa.it (M. d'Amore)
}

doi:10.3797/scipharm.cespt.8.PDD36

The drug release from solid matrices systems, made of polymes and drugs, is a basic concept for studies on controlled drug release. The most interesting class of polymers in this application is given by hydrogels. Matrices based on hydrogels, once swallowed (during the in-vitro tests, once immersed in the solvent mimicking body fluids), start to absorb water from the surrounding (water up-take) and the water causes a number of phenomena: the hydrogel swelling, the polymer plasticization (the lowering of the glass transition temperature), the increase of the diffusion coefficients, the polymer erosion (due to polymer chains disentanglement). As a result, the drug previously embedded in the matrix can be released (by diffusion into the matrix and then by dissolution in the outer solvent)

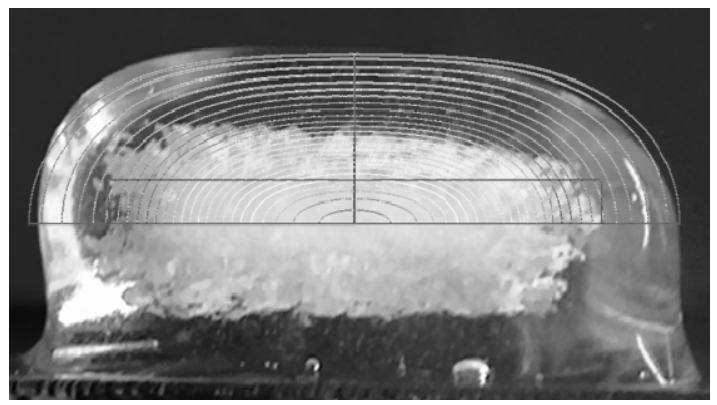

Fig. 1. Shape and hydration levels for the HPMC-TP 1:1 matrices subjected to the hydration. Snapshot of cut matrices (experimental) and superimposed simulated profiles (model) after 12 hours of hydration. The initial, non-swollen half-matrices shape is a rectangle (diameter $13 \mathrm{~mm}$, semi-thickness 1.1 $\mathrm{mm})$.

In this work, a model for the drug release kinetics was pointed out. Basically, it consists in differential balances of drug and water in the swelling matrices. It takes into account all the relevant phenomena: the diffusion of drug and water in polymer (with diffusion coefficients that vary with water concentration in the solid phase), the swelling and the dissolution of the polymer (which causes the boundary to be moving surfaces). The resulting code reveals itself able to correctly quantify all the observed phenomena, in terms of water, drug and polymer residual masses in the device, mass fractions of the three species, dimensions and shape of the matrix. To our knowledge, such a complete ability in description was never attained before. The model, therefore, could be a powerful tool in designing novel controlled drug release systems. 